\title{
Estimação da Oferta de Trabalho das Mulheres no Brasil
}

\author{
Ricardo Avelino \\ Naércio Menezes-Filho
}

Doutorando em Economia, University of Chicago

Professor da FEA-USP

\section{RESUMO}

Este artigo estima equações de oferta de trabalho para mulheres cônjuges no Brasil, no período entre 1992 e 1999, comparando três modelos existentes na literatura, o de StoneGeary, o de Heckman-MaCurdy e o de Blundell-Duncan-Meghir. Utiliza-se um método econométrico de pseudopainel, apropriado para cross-sections repetidas, no qual o agrupamento dos dados por coorte, tempo e escolaridade permite controlar o fenômeno estudado por características observáveis e não observáveis dos indivíduos. As elasticidades de oferta de trabalho encontradas variam entre -0,213 e 0,050, dependendo do modelo utilizado e da especificação escolhida para a oferta de trabalho.

\section{PALAVRAS-CHAVE}

oferta de trabalho, mulheres no mercado de trabalho, pseudopainel, cortes tranversais repetidos, análise de coorte

ABSTRACT

The paper estimates labor supply equations for married women in Brazil, between 1992 and 1999, comparing three existing models, Stone-Geary, Heckman-MaCurdy and Blundell-

Duncan-Meghir. An econometric method of pseudo panel is applied to repeated cross-sections, which allows the researcher to control for observed and unobserved characteristics of the individuals in the sample. The resulting elasticities are in the range of -0.213 to 0.050 , depending on the model and specification chosen for labor supply.

KEY WORDS

labor supply, women in the labor market, pseudo panel, repeated cross-sections, cohort analysis

JEL Classification

J22, J16 


\section{INTRODUÇÃO}

Uma das questóes cruciais de política econômica na área de economia do trabalho diz respeito às possíveis respostas do número de horas ofertado pelos trabalhadores em face de alterações nos salários. A magnitude e mesmo a direção da resposta é um tema controverso. Conforme ressaltado por Mroz (1987), diferenças nas suposições econômicas e estatísticas no que concerne a questóes como exogeneidade de variáveis, viés de seleção amostral, inclusão de impostos e erros de medida conduz a resultados completamente diversos.

O objetivo do presente trabalho é justamente analisar a elasticidade da oferta de trabalho feminina no Brasil com relação a modificações no salário. Não encontramos na literatura nenhuma estimativa de elasticidade de oferta de trabalho de mulheres usando dados brasileiros. Os estudos prévios nesta área estimaram a relação entre participação das mulheres e salários dos chefes de família, encontrando uma relação negativa entre estes dois fatores. (SEDLACEK \& SANTOS, 1991 e JATOBÁ, 1994). Ribeiro (1999) estimou a elasticidade de oferta dos homens brasileiros, encontrando estimativas negativas ou próximas a zero.

Seguindo estudos recentes, este artigo opta por uma perspectiva dinâmica ao analisar o problema no contexto do ciclo de vida, o que permite modelar as decisões de alocação intertemporais dos indivíduos por meio de técnicas de pseudopainel. Com o intuito de resolver o problema da limitação das informações fornecidas pelos dados no que se refere à trajetória completa das variáveis de interesse adota-se a sugestão proposta por MaCurdy (1981) de tratar a utilidade marginal da renda como um efeito fixo não observável.

A seção I, a seguir, apresenta uma descrição sucinta dos dados. São utilizados dados da Pesquisa Nacional por Amostra de Domicílios (PNAD), do IBGE, para 7 anos entre 1992 e 1999. ${ }^{1}$ É selecionada uma amostra de mulheres casadas entre 24 e 55 anos que não sejam chefes de família, devido ao fato de que esse grupo, mais do qualquer outro, apresenta grandes variaçóes no

1 Para o ano de 1994 não foi realizada a pesquisa. 
número de horas trabalhadas e é suficientemente flexível para responder a alterações no ambiente econômico.

Uma breve revisão da literatura no tocante a modelos de oferta de trabalho é apresentada na seção II, destacando-se dois modelos básicos derivados das funções de utilidade de Stone-Geary e Heckman-MaCurdy (aplicados por ANGRIST, 1991). A seção III expõe o modelo econométrico e discute algumas questões relativas à identificação, estimação e viés de seleção. Essa seção do trabalho está fortemente baseada em Blundell et al. (1998). A estratégia de identificação consiste em instrumentalizar as variáveis por coorte, educação e tempo, excluindo as interações entre essas três variáveis da equação principal. Com relação à estimação, utilizar-se-á a técnica de adição de resíduos, controlando também pelas possíveis mudanças de status oriundas da decisão de ofertar ou não trabalho por meio de um modelo probit.

Na seqüência, a seção IV apresenta o modelo para a determinação da oferta de trabalho das mulheres no Brasil, retoma rapidamente as técnicas de estimação empregadas e fornece os resultados das estimações realizadas, bem como das elasticidades. A última seção apresenta as conclusões do trabalho, destacando a comparação dos resultados obtidos com os de estudos prévios e possíveis extensões. A principal conclusão obtida é que a elasticidade da oferta de trabalho com relação ao salário é muito baixa e em alguns casos negativa.

\section{DESCRIÇÃO DOS DADOS}

Antes de passar à formalização do modelo econométrico utilizado, é conveniente fazer uma exposição sumária dos dados. ${ }^{2}$ A Tabela 1 apresenta o número de observações por ano, conforme a situação empregatícia das mulheres. Vale lembrar que estamos restringindo a amostra às mulheres cônjuges entre 24 e 55 anos de idade. Observa-se que a cada ano a amostra consiste de pouco menos de 40 mil pessoas, dentre as quais pouco menos da metade trabalha.

2 Os procedimentos utilizados para a construção da amostra são detalhados no apêndice. 


\section{TABELA I - NÚMERO DE OBSERVAÇÕES POR ANO CONFORME A} SITUAÇÃO DE EMPREGO

\begin{tabular}{lccc}
\hline Ano & Trabalha & Não trabalha & Total \\
\hline 1992 & 15557 & 19939 & 35496 \\
1993 & 16171 & 20113 & 36284 \\
1995 & 18740 & 20154 & 38894 \\
1996 & 17510 & 20854 & 38364 \\
1997 & 18731 & 21230 & 39961 \\
1998 & 18527 & 21038 & 39565 \\
1999 & 18880 & 19754 & 38634 \\
Total & 124116 & 143082 & 267198 \\
\hline
\end{tabular}

A Tabela 2 contempla a média de escolaridade por ano conforme o status no mercado de trabalho. Pode-se dizer que, em média, as mulheres que trabalham possuem dois anos a mais de estudo do que as que não trabalham e que a escolaridade média para o conjunto das mulheres vem aumentando a partir de 1993. É de se supor que os dois grupos difiram não só em variáveis observáveis como também em características não observáveis.

TABELA 2 - MÉDIA DE ANOS DE EDUCAÇÃO POR ANO CONFORME A SITUAÇÃO DE EMPREGO (DESVIOS PADRÕES ENTRE PARENTESES)

\begin{tabular}{lrrr}
\hline Ano & Trabalha & Não trabalha & \multicolumn{1}{c}{ Média } \\
\hline 1992 & 8,01838 & 6,1210 & 6,9526 \\
& $(4,7349)$ & $(3,8606)$ & $(4,3685)$ \\
1993 & 8,0907 & 6,2104 & 7,0484 \\
& $(4,7065)$ & $(3,8577)$ & $(4,3583)$ \\
1995 & 8,2052 & 6,3368 & 7,2370 \\
& $(4,6119)$ & $(3,8566)$ & $(4,3390)$ \\
1996 & 8,4148 & 6,4284 & 7,3350 \\
& $(4,5753)$ & $(3,8777)$ & $(4,3251)$ \\
1997 & 8,4722 & 6,4573 & 7,4017 \\
& $(4,5643)$ & $(3,8906)$ & $(4,3379)$ \\
1998 & 8,6088 & 6,6338 & 7,5586 \\
& $(4,5252)$ & $(3,8976)$ & $(4,3171)$ \\
1999 & 8,5991 & 6,8025 & 7,6805 \\
& $(4,4957)$ & $(3,9390)$ & $(4,3147)$ \\
Média & 8.3569 & 6.4281 & 7,3241 \\
& $(4.6025)$ & $(3.8889)$ & $(4,3432)$ \\
\hline
\end{tabular}


A correlação entre as variáveis para as mulheres que ofertam um número de horas superior a zero é apresentada na Tabela 3. A variável renda corresponde ao rendimento mensal familiar de todos os moradores, exclusive o salário de todas as fontes da mulher. Já a variável educação foi gerada de forma que seja igual a um se as mulheres possuem ensino fundamental completo e zero em caso contrário. ${ }^{3}$ Enquanto isso, horas representa o número de horas semanais trabalhadas para as mulheres engajadas num trabalho remunerado durante a semana de referência e salário corresponde ao rendimento mensal de todos os trabalhos dividido pelo número de horas trabalhadas. ${ }^{4} \mathrm{~A}$ variável coorte assume seis valores distintos de acordo com a faixa em que a data de nascimento dos indivíduos está compreendida: 1937-43, 1944-50, 1951-57, 1958-63, 1964-1969 e 1970-75.

Um aspecto interessante dos dados é a correlação negativa entre horas e salário, pois a teoria econômica prevê uma elasticidade intertemporal positiva da oferta de trabalho. Entretanto, baseando-se apenas nessa tabela, não é possível determinar, ceteris paribus, os efeitos de uma elevação hipotética dos salários no número de horas ofertado. A decisão das mulheres de trabalhar um maior ou menor número de horas provavelmente também é influenciada por outras características observáveis como, por exemplo, educação e renda familiar, que são positivamente correlacionadas com o salário, e por características não observáveis, como habilidade, criatividade e dinamismo, entre outras.

3 Na construção dos pseudopainéis, detalhada na seção III, requer-se que o número de observaçôes por célula tenda a infinito para que os estimadores dos parâmetros sejam consistentes. As células são definidas por diferentes valores das variáveis educação, coorte e ano. Como a utilização da educação na forma contínua acarretaria um número muito pequeno de observações em algumas células, sobretudo para aquelas que incluem os indivíduos mais instruídos, optouse por transformá-la em discreta.

4 Os valores monetários foram ajustados para reais de setembro de 1999, utilizando o deflator proposto por Corseuil \& Fogel. 


\section{TABELA 3 - CORRELAÇÃO ENTRE AS VARIÁVEIS}

\begin{tabular}{lrrrrrrr}
\hline Variáveis & Horas & Salário & Renda & Idade & Educação & Ano & Coorte \\
\hline Horas & 1,0000 & & & & & & \\
Salário & $-0,1343$ & 1,0000 & & & & & \\
Renda & $-0,0057$ & 0,3840 & 1,0000 & & & & \\
Idade & $-0,0215$ & 0,0322 & 0,1011 & 1,0000 & & & \\
Educação & 0,0058 & 0,2629 & 0,2605 & $-0,1632$ & 1,0000 & & \\
Ano & 0,0306 & 0,0341 & 0,0265 & 0,0389 & 0,0490 & 1,0000 & \\
Coorte & 0,0282 & $-0,0233$ & $-0,0875$ & $-0,9321$ & 0,1669 & 0,2466 & 1,0000 \\
\hline
\end{tabular}

Pode-se notar também uma correlação positiva entre salário e educação, conforme previsto pela teoria econômica, e entre educação e renda. Observa-se ainda uma correlação negativa entre idade e educação, o que sugere que nas últimas décadas tem aumentado a qualificação requerida pelo mercado de trabalho e ocorrido uma disseminação do acesso à educação para as classes menos favorecidas da população.

\section{MODELOS DE OFERTA DE TRABALHO}

O primeiro modelo a ser considerado é derivado da função de utilidade de Stone-Geary. ${ }^{5}$ Num contexto intertemporal, o consumidor representativo depara-se com o seguinte problema de otimização: ${ }^{6}$

$$
\operatorname{Max} \mathrm{v}=\sum_{t} \frac{1}{(1+\rho)^{t}}\left[B_{1} \ln \left(\gamma-\mathrm{h}_{\mathrm{t}}\right)+B_{2} \ln \left(\mathrm{c}_{\mathrm{t}}-\zeta\right)\right]
$$

sujeito a

$$
\sum_{t} \frac{1}{(1+r)^{t}}\left[\mathrm{w}_{\mathrm{t}} \mathrm{h}_{\mathrm{t}}+\mathrm{y}_{\mathrm{t}}-\mathrm{c}_{\mathrm{t}}\right]=0
$$

5 Ver ASHENFELTER \& HAM (1979).

6 Sem perda de generalidade, pode-se normalizar um dos preços em cada período. No que se segue, optou-se por adotar um preço unitário para o bem de consumo composto. Desse modo, a variável salário corresponde ao salário real. 
$\mathrm{Na}$ função (l) acima, aditiva tanto no tempo quanto no consumo e no lazer, $\mathrm{h}_{\mathrm{t}}$ é o número de horas ofertado, $\mathrm{c}_{\mathrm{t}}$ a quantidade de consumo escolhida, $\mathrm{w}_{\mathrm{t}} \mathrm{o}$ salário, $\mathrm{y}_{\mathrm{t}}$ simboliza as outras rendas familiares, $\rho$ a taxa de desconto e $\mathrm{r}$ a taxa de juros. Enquanto isso, $B_{1}, B_{2}, \gamma$ e $\zeta$ são parâmetros a serem estimados. Os parâmetros $\gamma$ e $\zeta$ possuem uma interpretação econômica conveniente. O primeiro representa o número máximo de horas de trabalho factível. Já o segundo corresponde ao consumo mínimo de bens necessário. Das condições de primeira ordem, após algumas substituições, segue-se que: ${ }^{7}$

$$
\mathrm{w}_{\mathrm{t}} \mathrm{h}_{\mathrm{t}}=\gamma \mathrm{w}_{\mathrm{t}}-\eta^{\mathrm{t}} \tilde{B}_{1} F^{*}
$$

em que:

$$
\begin{aligned}
& \eta=(1+\mathrm{r}) /(1+\rho), \tilde{B}_{1}=B_{1} / \sum_{t}\left(B_{1 t} *+B_{2 t} *\right), F^{*}=\sum_{t}\left[\mathrm{w}{ }_{\mathrm{t}}^{*}+\mathrm{y}_{\mathrm{t}} * \varsigma \mathrm{p}_{\mathrm{t}}^{*}\right], \\
& B_{i t} *=B_{i}(1+\rho)^{-\mathrm{t}}, \quad \mathrm{w}_{\mathrm{t}}^{*}=\mathrm{w}_{\mathrm{t}}(1+\mathrm{r})^{-\mathrm{t}}, \mathrm{y}_{\mathrm{t}}^{*}=\mathrm{y}_{\mathrm{t}}(1+\mathrm{r})^{-\mathrm{t}}, \mathrm{p}_{\mathrm{t}}^{*}=(1+\mathrm{r})^{-\mathrm{t}}
\end{aligned}
$$

O segundo modelo utilizado é proveniente do trabalho de Heckman e MaCurdy (1980). Neste modelo, os consumidores supostamente possuem um horizonte de tempo finito $\mathrm{T}$ e a função de utilidade num instante de tempo t é fortemente côncava e expressa por $\mathrm{G}(\mathrm{C}(\mathrm{t}))+\mathrm{J}(\mathrm{L}(\mathrm{t}))$. $\mathrm{C}(\mathrm{t})$ e $\mathrm{L}(\mathrm{t})$ são, respectivamente, as quantidades instantâneas de consumo de bens e lazer. Uma normalização conveniente é assumir que $0 \leq \mathrm{L}(\mathrm{t}) \leq 1$. Além disso, os indivíduos deparam-se com um salário $W_{t}$ independente de suas escolhas. As variáveis $\mathrm{y}_{\mathrm{t}}, \mathrm{p}_{\mathrm{t}}, \rho$ e r representam, respectivamente, a riqueza inicial, $\mathrm{o}$ nível de preços, a taxa de desconto e a taxa de juros. Por simplicidade, o nível de preços é normalizado a um novamente. Portanto, o problema do consumidor é:

7 Detalhes da derivação da equação (3) são fornecidos no apêndice.

8 Conforme notado por ASHENFELTER \& HAM (1979), essa função faz parte da classe mais ampla de funções denominadas por HECKMAN \& MACURDY (1980) de " $\lambda$ constante lifecycle supply functions". 


$$
\operatorname{Max} \int_{o}^{T} e^{-\rho t}[\mathrm{G}(\mathrm{C}(\mathrm{t}))+\mathrm{J}(\mathrm{L}(\mathrm{t}))] \mathrm{dt}
$$

sujeito a

$$
\mathrm{y}_{\mathrm{t}}=\int_{0}^{T} e^{-t}[\mathrm{C}(\mathrm{t})-\mathrm{W}(\mathrm{t})(1-\mathrm{L}(\mathrm{t}))] \mathrm{dt}
$$

As condições para obtenção de um ótimo são dadas por:

$$
\begin{aligned}
& \mathrm{G}^{\prime}(\mathrm{C}(\mathrm{t}))=e^{(\rho-r) t} \lambda(0) \\
& \mathrm{J}^{\prime}(\mathrm{L}(\mathrm{t})) \geq e^{(\rho-r) t} \lambda(0) \mathrm{W}_{\mathrm{t}} \\
& \lambda(\mathrm{t})=e^{-t} \lambda(0)
\end{aligned}
$$

Nas equações acima, $\lambda(0)$ é o multiplicador de Lagrange associado à restrição orçamentária e, dada a normalização adotada para o lazer, a utilidade marginal da riqueza no período 0 . Adotando uma forma funcional simples para a função utilidade, semelhante à de Heckman e MaCurdy (1980), e trabalhando com intervalos de tempo discretos, substituiu-se a função objetivo por:

$$
\sum_{o}^{T} \frac{1}{(1+\rho)^{t}}\left[\mathrm{~A}(\mathrm{t})(\mathrm{L}(\mathrm{t}))^{\alpha}+\mathrm{B}(\mathrm{t})(\mathrm{C}(\mathrm{t}))^{\beta}\right]
$$

$\mathrm{A}(\mathrm{t})$ e $\mathrm{B}(\mathrm{t})$ são parâmetros específicos ao indivíduo e podem variar no tempo, deslocando as preferências por lazer e consumo. A função de demanda derivada da maximização de utilidade por parte do consumidor é dada, se o indivíduo trabalha, por: ${ }^{9}$

$\mathrm{L}(\mathrm{t})=\left[\frac{1}{A(t) \alpha}\left(\frac{1+\rho}{1+r}\right)^{t} \lambda(0) W(t)\right]^{\frac{1}{\alpha-1}}$

9 Ver apêndice. 
Se o indivíduo não trabalha:

$$
\mathrm{L}(\mathrm{t})=\bar{L}
$$

Aplicando logaritmo a ambos os lados de (10a), tem-se que:

$\ln (\bar{L}-\mathrm{h}(\mathrm{t}))=1 /(\alpha-1)[\ln \lambda(0)-\ln \alpha+(\rho-\mathrm{r}) \mathrm{t}-\ln \mathrm{A}(\mathrm{t})+\ln \mathrm{W}(\mathrm{t})](11)$

Alternativamente, (11) pode ser expressa como:

$\ln (\bar{L}-\mathrm{h}(\mathrm{t}))=1 / \alpha[\ln \lambda(0)-\ln \alpha+(\rho-\mathrm{r}) \mathrm{t}-\ln \mathrm{A}(\mathrm{t})+\ln \mathrm{W}(\mathrm{t}) \mathrm{h}(\mathrm{t})]$

Finalmente, substituindo as funções de demanda (6) e (7) na restrição orçamentária (5), pode-se expressar $\lambda(0)$ como uma função implícita do salário, da quantidade inicial de ativos, da taxa de juros e da taxa de desconto.

\section{MODELO ECONOMÉTRICO}

Os modelos de oferta de trabalho oriundos de teorias do ciclo de vida trazem consigo a complicação de que as decisões em cada instante são funções não só de preços e salários contemporâneos como também dos valores dessas variáveis em todos os períodos.

Objetivando solucionar esse problema, adotar-se-á a solução proposta por MaCurdy (1981). As equações (3), (11) e (12) mostram que a decisão de se ofertar trabalho num determinado instante de tempo t é função de variáveis não observadas contemporaneamente somente por meio de $\lambda(0)$ $\left(\mathrm{F}^{*}\right)$. Como $\lambda(0)\left(\mathrm{F}^{*}\right)$ é constante para o indivíduo ao longo do ciclo de vida, pode ser tratado como um efeito fixo, reduzindo-se a dimensão do problema. Qualquer trajetória dos salários ou da renda ao longo do ciclo de vida que mantenha inalterado $\lambda(0)\left(\mathrm{F}^{*}\right)$ e o valor corrente do salário implica a mesma decisão ótima de consumo e lazer em um dado instante. 
As subseções seguintes descrevem sucintamente o modelo de efeitos fixos, as condições necessárias para sua identificação, estratégias de estimação e os problemas ocasionados pelo viés de seleção.

\section{III.1 Modelo de Efeitos Fixos}

A equação de interesse pode ser resumida da seguinte forma:

$$
\begin{aligned}
& \mathrm{y}_{\mathrm{i}(\mathrm{t}) \mathrm{t}}=\beta_{0}+\beta_{1} \mathrm{x}_{\mathrm{i}(\mathrm{t}) \mathrm{t}}+\beta_{2} \mathrm{z}_{\mathrm{i}(\mathrm{t})}+\mathrm{f}_{\mathrm{i}(\mathrm{t})}+\sum_{t=1}^{T} \varphi_{\mathrm{t}} \gamma_{\mathrm{t}}+\varepsilon_{\mathrm{i}(\mathrm{t}) \mathrm{t}} \\
& \mathrm{i}(\mathrm{t})=1,2, \ldots \ldots, \mathrm{N} \quad \mathrm{t}=1,2, \ldots \ldots ., \mathrm{T} .
\end{aligned}
$$

Na equação (14), $\mathrm{y}_{\mathrm{i}(\mathrm{t}) \mathrm{t}}$ é a variável dependente, $\mathrm{x}_{\mathrm{i}(\mathrm{t}) \mathrm{t}}$ é um conjunto de regressores que variam no tempo, $\mathrm{z}_{\mathrm{i}(\mathrm{t})}$ são regressores invariantes no tempo, $\mathrm{f}_{\mathrm{i}(\mathrm{t})}$ representa o efeito fixo não observável, inerente a cada indivíduo, possivelmente correlacionado com $\mathrm{x}_{\mathrm{i}(\mathrm{t}) \mathrm{t}}$, e $\varepsilon_{\mathrm{i}(\mathrm{t})}$ é um erro não observável que, por hipótese, é ortogonal a $\mathrm{x}_{\mathrm{i}(\mathrm{t}) \mathrm{t}}, \mathrm{z}_{\mathrm{i}(\mathrm{t})} \mathrm{e}_{\mathrm{i}(\mathrm{t})}$. Com o intuito de captar as variações temporais comuns a todas as pessoas em dado período é colocado um conjunto de variáveis binárias $\gamma_{\mathrm{t}}$ que assumem o valor 1 se $\mathrm{t}=\mathrm{t}_{\mathrm{i}} \mathrm{e} 0 \mathrm{em}$ caso contrário. $\beta_{0}, \beta_{1}, \beta_{2} \mathrm{e} \varphi_{\mathrm{t}}$ são parâmetros a serem estimados.

\section{III.2 Identificação}

Como $\mathrm{f}_{\mathrm{i}(\mathrm{t})}$ inclui $\lambda(0)$ como um de seus componentes $\lambda(0)\left(\mathrm{F}^{*}\right)$ e $\lambda(0)\left(\mathrm{F}^{*}\right)$ depende do valor das variáveis e das restrições em todos os períodos, $\mathrm{x}_{\mathrm{i}(\mathrm{t}) \mathrm{t}} \mathrm{e}$ $\mathrm{f}_{\mathrm{i}(\mathrm{t})}$ são correlacionados. Portanto, a estimação da equação (14) por mínimos quadrados ordinários produz estimadores inconsistentes, já que $f_{\mathrm{i}(\mathrm{t})}$ é absorvido pelo erro na estimação. Uma dificuldade adicional na identificação desse tipo de modelo advém da ausência de observações passadas para os indivíduos observados num dado instante de tempo.

Assim, para obter estimadores consistentes é necessário recorrer ao método de variáveis instrumentais. ${ }^{10}$ A classe mais importante de instrumentos con-

10 Uma discussão detalhada com respeito à identificação do modelo de efeitos fixos no caso de $\operatorname{cross}$-sections repetidas é realizada por MOFFITT (1993). 
siste de funções do tempo, que certamente não são correlacionadas com $\mathrm{f}_{\mathrm{i}(\mathrm{t})}$. Além disso, se houver efeitos de coorte na população, como freqüentemente ocorre em estudos comportamentais, estimadores mais eficientes podem ser obtidos se levarmos em conta as características individuais. Para tanto, o ano de nascimento pode ser utilizado como instrumento. Consideremos a seguinte projeção linear de $\mathrm{x}_{\mathrm{i}(\mathrm{t}) \mathrm{t}}$, na qual $\mathrm{g}_{\mathrm{lk}}$ são funções do tempo, $\mathrm{g}_{2 \mathrm{~m}}$ são funções dos coortes e $g_{3 j}$ são interações entre tempo e coorte.

$$
\mathrm{x}_{\mathrm{i}(\mathrm{t}) \mathrm{t}}=\sum_{k=1}^{K} \delta_{1 \mathrm{k}} \mathrm{g}_{1 \mathrm{k}}(\mathrm{t})+\sum_{m=1}^{M} \delta_{2 \mathrm{~m}} \mathrm{~g}_{2 \mathrm{~m}}\left(\mathrm{c}_{\mathrm{i}(\mathrm{t})}\right)+\sum_{j=1}^{J} \delta_{3 \mathrm{j}} \mathrm{g}_{3 \mathrm{j}}\left(\mathrm{c}_{\mathrm{i}(\mathrm{t})}, \mathrm{t}\right)+\mathrm{u}_{\mathrm{i}(\mathrm{t}) \mathrm{t}}
$$

Denotemos por $x_{t}$ os valores da equação (15) previstos por mínimos quadrados ordinários. A consistência do estimador de variáveis instrumentais requer que:

i) $x_{t}$ varie com o tempo

ii) $\operatorname{plim}\left[(\mathrm{l} / \mathrm{NT}) \sum_{i(t), t} \hat{x}_{t} \mathrm{f}_{\mathrm{i}(\mathrm{t})}=0\right.$

Um instrumento baseado em efeitos de coorte, entretanto, provavelmente estará correlacionado com $\mathrm{f}_{\mathrm{i}(\mathrm{t})}$. Sendo assim, a segunda condição não é satisfeita. Definamos, todavia, $\bar{f}_{c(t)}$ como o efeito fixo médio dos indivíduos observados no instante $\mathrm{t}$ e nascidos no ano $\mathrm{c}$. Considerando $\mathrm{v}_{\mathrm{i}(\mathrm{t})}$ como o desvio de $\mathrm{f}_{\mathrm{i}(\mathrm{t})}$ com relação à média amostral $\bar{f}_{c(t)} \mathrm{e} \mathrm{v}_{{ }^{\prime}(\mathrm{t})}$ como o desvio da média amostral com relação à média populacional $\bar{f}_{c}$ *, temos que:

$$
\mathrm{f}_{\mathrm{i}(\mathrm{t})}=\bar{f}_{c} *+\mathrm{v}_{\mathrm{c}(\mathrm{t})}+\mathrm{v}_{\mathrm{i}(\mathrm{t}) \mathrm{t}}
$$

Claramente, se supusermos que a população é fechada, $\bar{f}_{c} *$ será constante. Como $\bar{f}_{c} *$ pode ser representado explicitamente na equação (14) por dummies de coorte, a segunda condição requer simplesmente que $x_{t}$ não seja assintoticamente correlacionado com $v_{c(t)}^{\prime}{ }^{\prime} v_{i(t) t}$. Mas quando $N \rightarrow \infty$ e o número de coortes é mantido fixo, o erro amostral $v^{\prime}{ }_{c(t)}$ tende a zero e os desvios individuais $\mathrm{v}_{\mathrm{i}(\mathrm{t}) \mathrm{t}}$ passam a não depender de $\mathrm{t} \mathrm{e}$, portanto, de $x_{t}$, pois a amostra torna-se idêntica à população. 
É importante ressaltar, contudo, que, de modo geral, as funções $g_{2 m}$ não possuem, necessariamente, apenas os coortes como argumento. Qualquer conjunto de características invariantes no tempo como, por exemplo, sexo, raça e educação (se ignorarmos a possibilidade dos indivíduos retomarem os estudos) pode ser incluído.

\section{III.3 Estimação}

Sejam $\overline{x_{c t}}$ a média de $\mathrm{x}_{\mathrm{i}(\mathrm{t}) \mathrm{t}}$ do coorte $\mathrm{c}$ no instante de tempo $\mathrm{t}, \overline{x_{c}}$ a média de $\mathrm{x}_{\mathrm{i}(\mathrm{t}) \mathrm{t}}$ do coorte c e $\bar{x}_{t}$ a média de $\mathrm{x}_{\mathrm{i}(\mathrm{t}) \mathrm{t}}$ no instante de tempo t. Definamos $\overline{\overline{x_{c t}}}=\overline{x_{c t}}-\overline{x_{c}}-\overline{x_{t}}$. Similarmente, definamos $\overline{\overline{y_{c t}}}$ e $\overline{\overline{z_{c t}}}$. Consideremos, ainda, $\mathrm{n}_{\mathrm{ct}}$ como o número de observações da célula $(\mathrm{c}, \mathrm{t})$. Uma das alternativas para estimar o modelo proposto acima é utilizar o estimador de Wald, que pode ser expresso da seguinte forma para os coeficientes de $\mathrm{x}_{\mathrm{i}(\mathrm{t}) \mathrm{t}}$ :

$$
\hat{b}=\frac{\sum_{c} \sum_{t}\left[\overline{\overline{y_{c t}}}\right]\left[\overline{\overline{x_{c t}}}\right] / n_{c t}}{\sum_{c} \sum_{t}\left(\overline{\overline{x_{c t}}}\right)^{2} / n_{c t}}
$$

Analogamente, pode-se defini-lo para os coeficientes de $\mathrm{z}_{\mathrm{i}(\mathrm{t})}$. Conforme notado por Blundell et al. (1998), uma forma alternativa de se obter a estimativa dos parâmetros e que produz resultados numericamente idênticos é via técnica de adição de resíduos. Primeiramente, para implementar esse estimador, deve-se estimar por mínimos quadrados ordinários uma regressão de $\mathrm{x}_{\mathrm{i}(\mathrm{t}) \mathrm{t}}$ contra um conjunto de interações de coorte e tempo ${ }^{11} \mathrm{e}$ calcular os resíduos. Em seguida, regride-se $\mathrm{y}_{\mathrm{i}(\mathrm{t}) \mathrm{t}}$ contra $\mathrm{x}_{\mathrm{i}(\mathrm{t}) \mathrm{t}}$ e $\mathrm{z}_{\mathrm{i}(\mathrm{t}) \mathrm{t}}$, dummies de coorte, dummies de ano e os resíduos calculados no primeiro estágio. Esse método apresenta a vantagem de fornecer testes diretos de exogeneidade para as variáveis explicativas com base nas estatísticas $t$ calculadas para os coeficientes dos resíduos. ${ }^{12}$

11 Além disso, como mencionado anteriormente, pode-se aumentar o número de interações incluindo-se regressores invariantes no tempo específicos a cada indivíduo.

12 Os desvios padrões estimados dos resíduos precisam apenas ser corrigidos devido ao fato dos valores dessas variáveis utilizados no segundo estágio serem valores estimados. 


\section{III.4 Viés de Seleção}

Suponhamos que a amostra possa ser dividida em dois grupos, conforme as pessoas decidam ofertar ou não trabalho. Ao estimar o modelo, devemos estar cientes de que estamos implicitamente fazendo a suposição de que a esperança do erro, condicional no fato das mulheres ofertarem trabalho, pode ser representada de maneira adequada por um efeito coorte $\left(a_{c}\right)$ e um efeito tempo $\left(m_{t}\right)$, de forma aditiva: ${ }^{13}$

$$
E\left[u_{i t} \mid P_{i t}, c, t\right]=a_{c}+m_{t}
$$

em que $\mathrm{P}_{\text {it }}$ indica que o indivíduo é observado trabalhando. Em outras palavras, as diferenças entre os grupos, condicionais nos regressores, são invariantes no tempo. Além disso, as variáveis explicativas devem crescer de forma diferente entre os grupos ao longo do tempo, o que equivale à condição de posto para identificação:

$$
\mathrm{E}\left[\overline{\overline{x_{c t}}}\right]^{2} \neq 0
$$

No entanto, a hipótese (17) pode ser violada caso a participação não esteja sendo determinada de forma aleatória. Suponhamos, por exemplo, que a variável de interesse seja uma variável latente $\mathrm{y}_{\mathrm{i}}^{*}=\beta \mathrm{x}_{\mathrm{i}}+\mathrm{u}_{\mathrm{i}}$ tal que

$$
\begin{aligned}
& \mathrm{y}_{\mathrm{i}}=\mathrm{y}_{\mathrm{i}}^{*} \text { se } \mathrm{S}_{\mathrm{i}}>0 \text { e } \\
& \mathrm{y}_{\mathrm{i}}=0 \quad \text { se } \mathrm{S}_{\mathrm{i}}<0, \\
& \mathrm{~S}_{\mathrm{i}}=\eta \mathrm{z}_{\mathrm{i}}+\varepsilon_{\mathrm{i}}
\end{aligned}
$$

Um resultado estabelecido na literatura nos diz que ${ }^{14}$

$$
\mathrm{E}\left[\mathrm{y}_{\mathrm{i}} \mid \mathrm{x}_{\mathrm{i}}, \mathrm{z}_{\mathrm{i}}, \mathrm{S}_{\mathrm{i}}>0\right]=\beta \mathrm{x}_{\mathrm{i}}+\rho \lambda\left(\eta \mathrm{z}_{\mathrm{i}} / \varepsilon_{\mathrm{i}}\right) / \sigma_{\mathrm{U}}
$$

13 Ver BLUNDELL et al. (1998)

14 Ver HECKMAN (1979). 
em que $\rho$ é a correlação entre $\mathrm{u}_{\mathrm{i}}$ e $\varepsilon_{\mathrm{i}}, \sigma_{\mathrm{U}} \mathrm{o}$ desvio padrão de $\mathrm{u}_{\mathrm{i}}$ e $\lambda$ a razão de Mills invertida avaliada no ponto $\eta \mathrm{z}_{\mathrm{i}} / \varepsilon_{\mathrm{i}}$. Portanto, se ignorarmos o mecanismo de seleção, estaremos incorrendo num viés de variável omitida. Para sanar esse problema, pode-se estender (17) e (18) de modo que:

$$
\begin{aligned}
& \mathrm{E}\left[\mathrm{u}_{\mathrm{it}} \mid \mathrm{P}_{\mathrm{it}}, \mathrm{c}, \mathrm{t}\right]=\mathrm{a}_{\mathrm{c}}+\mathrm{m}_{\mathrm{t}}+\xi \lambda_{\mathrm{ct}} \\
& \mathrm{E}\left[\overline{\overline{x_{c t}}}-\zeta_{\mathrm{x}} \lambda_{\mathrm{ct}}\right]^{2} \neq 0
\end{aligned}
$$

Em (18') $\zeta_{x}$ é o coeficiente de correlação parcial populacional definido por $\zeta_{\mathrm{x}}=\mathrm{E}\left[\overline{\overline{x_{c t}}} \overline{\overline{\lambda_{c t}}}\right] / \mathrm{E}\left[\overline{\overline{\lambda_{c t}}}\right]^{2}, \overline{\overline{\lambda_{c t}}}$ é definido analogamente a $\overline{\overline{x_{c t}}}$. Como discutido na seção III.3, esse estimador pode ser implementado utilizando uma técnica de adição de resíduos. Primeiramente, deve-se estimar por mínimos quadrados ordinários uma regressão de $\mathrm{x}_{\mathrm{i}(\mathrm{t}) \mathrm{t}}$ contra um conjunto de interações de coorte e tempo e calcular os resíduos. Em seguida, regridese $\mathrm{y}_{\mathrm{i}(\mathrm{t}) \mathrm{t}}$ contra $\mathrm{x}_{\mathrm{i}(\mathrm{t}) \mathrm{t}}$ e $\mathrm{z}_{\mathrm{i}(\mathrm{t}) \mathrm{t}}$, dummies de coorte, dummies de ano e os resíduos calculados no primeiro estágio, além da razão de Mills invertida calculada a partir da estimação de um probit para toda a amostra, objetivando modelar o mecanismo de seleção.

\section{OFERTA DE TRABALHO DAS MULHERES NO BRASIL}

\section{IV.1 Modelos}

O primeiro modelo proposto para investigar a sensibilidade da oferta de trabalho das mulheres no Brasil é expresso pela equação (20). Deriva-se, essencialmente, da função de utilidade de Stone-Geary, sendo adaptado para um contexto de cortes transversais repetidos.

$$
\mathrm{w}_{\mathrm{i}(\mathrm{t}) \mathrm{h}} \mathrm{h}_{\mathrm{i}(\mathrm{t}) \mathrm{t}}=\beta_{1} \mathrm{~W}_{\mathrm{i}(\mathrm{t}) \mathrm{t}}+\beta_{2} \mathrm{v}_{\mathrm{i}(\mathrm{t}) \mathrm{t}}(\mathrm{w})+\sum_{j=3}^{6} \beta_{\mathrm{j}} \mathrm{DK}_{\mathrm{i}(\mathrm{t}) \mathrm{t}}+\sum_{k=1}^{12} \delta_{\mathrm{k}} \mathrm{ce}_{\mathrm{i}(\mathrm{t})}+\sum_{t=1}^{8} \varphi_{\mathrm{t}} \gamma_{\mathrm{t}}+\zeta \lambda_{\mathrm{i}(\mathrm{t}) \mathrm{t}}+\varepsilon_{\mathrm{i}(\mathrm{t}) \mathrm{t}}
$$


Na equação anterior, $\mathrm{h}_{\mathrm{i}(\mathrm{t}) \mathrm{t}}$ representa o número de horas de trabalho mensal do indivíduo i no instante $\mathrm{t}, \mathrm{w}_{\mathrm{i}(\mathrm{t}) \mathrm{t}}$ é o respectivo salário, $\mathrm{DK}_{\mathrm{i}(\mathrm{t}) \mathrm{t}}$ são variáveis demográficas que indicam a idade do filho mais novo, conforme discutido a seguir, e $\lambda_{\mathrm{i}(\mathrm{t}) \mathrm{t}}$ é a razão de Mills invertida, incluída para captar o processo de seleção. Já ce $_{\mathrm{i}(\mathrm{t})}$ e $\gamma_{\mathrm{t}}$ são, respectivamente, dummies geradas a partir de interações entre coorte e educação e dummies de ano. Por fim, $\mathrm{v}_{\mathrm{i}(\mathrm{t}) \mathrm{t}}{ }^{(\mathrm{w})}$ é o resíduo correspondente ao salário.

O primeiro passo na estimação da oferta de trabalho das mulheres no Brasil consiste na seleção dos regressores. Trabalhos anteriores sugerem que o número de filhos e sua faixa etária são um importante determinante do número de horas ofertado pelas mulheres. ${ }^{15}$ Dessa maneira, optou-se por considerar $\tilde{B}_{1}$ na equação (3) como função de variáveis dummy, denotadas por DK02, DK34, DK510 e DK11. Estas variáveis assumem o valor um caso o filho mais novo tenha entre 0 e 2,3 ou 4 , entre 5 e 10 ou 11 anos ou mais, respectivamente.

Adicionalmente, incluíram-se dummies de tempo para captar diferenças entre a taxa de juros e a taxa de desconto, representada pelo termo $\eta^{t}$ na equação (3). Já as dummies de coorte e educação são incluídas para eliminar a correlação do efeito fixo, representado por $\mathrm{F}^{*}$ na equação (3), com o erro $\varepsilon_{\mathrm{i}(\mathrm{t}) \mathrm{t}}$, conforme discutido na seção III. ${ }^{16}$

15 Ver, por exemplo, HECKMAN \& MACURDY (1980), MACURDY (1981), MROZ (1987) e BLUNDELL et al. (1998).

16 É indispensável ressaltar que se optou por não incluir polinômios envolvendo a idade como uma proxy para experiência para evitar multicolinearidade, porque, sabendo-se o coorte do indivíduo e a data em que ele foi observado, é possível determinar sua faixa etária. Uma vantagem dessa especificação é que ela impõe uma forma funcional flexível para a idade, uma vez que esta é incorporada indiretamente por meio de dummies de coorte e tempo, cujos coeficientes podem variar de um período para outro. 
A equação (21) apresenta o segundo modelo considerado, baseado na função de utilidade utilizada por Heckman e MaCurdy (1980). Esse modelo é derivado de um problema de maximização bastante similar. Difere da equação (20) basicamente pelo fato de regredir o logaritmo do número de horas trabalhadas contra o logaritmo do salário, em vez dos ganhos totais contra o salário. Os parâmetros $\mathrm{A}(\mathrm{t})$ e $\mathrm{B}(\mathrm{t})$, que deslocam as preferências por consumo e lazer, foram considerados função do número de filhos e de sua faixa etária, assim como $\tilde{B}_{1}$. Já a variável $\mathrm{v}_{\mathrm{i}(\mathrm{t}) \mathrm{t}}{ }^{(\ln \mathrm{w})}$ representa o resíduo associado à $\ln \mathrm{w}_{\mathrm{i}(\mathrm{t})}$.

$$
\begin{aligned}
& \ln \mathrm{h}_{\mathrm{i}(\mathrm{t}) \mathrm{t}}=\beta_{0}+\beta_{1} \ln \mathrm{w}_{\mathrm{i}(\mathrm{t}) \mathrm{t}}+\beta_{2} \mathrm{v}_{\mathrm{i}(\mathrm{t}) \mathrm{t}}(\ln \mathrm{w})+\sum_{j=3}^{6} \beta_{\mathrm{j}} \mathrm{DK}_{\mathrm{i}(\mathrm{t}) \mathrm{t}}+\sum_{k=1}^{12} \delta_{\mathrm{k}} \mathrm{ce}_{\mathrm{i}(\mathrm{t})}+ \\
& \sum_{t=1}^{8} \varphi_{\mathrm{t}} \gamma_{\mathrm{t}}+\zeta \lambda_{\mathrm{i}(\mathrm{t}) \mathrm{t}}+\varepsilon_{\mathrm{i}(\mathrm{t}) \mathrm{t}}
\end{aligned}
$$

Enquanto isso, a equação (22) retrata simplesmente o modo alternativo de se estimar o modelo de Heckman e MaCurdy, regredindo o logaritmo do salário contra o logaritmo da renda, tal como destacado na seção II. A variável $\mathrm{v}_{\mathrm{i}(\mathrm{t}) \mathrm{t}}(\ln w \mathrm{~h})$ representa o resíduo associado à $\ln \mathrm{w}_{\mathrm{i}(\mathrm{t}) \mathrm{t}} \mathrm{h}_{\mathrm{i}(\mathrm{t}) \mathrm{t}}$, estimado no primeiro estágio.

$$
\begin{aligned}
& \ln \mathrm{h}_{\mathrm{i}(\mathrm{t}) \mathrm{t}}=\beta_{0}+\beta_{1} \ln \mathrm{w}_{\mathrm{i}(\mathrm{t}) \mathrm{t}} \mathrm{h}_{\mathrm{i}(\mathrm{t}) \mathrm{t}}+\beta_{2} \mathrm{v}_{\mathrm{i}(\mathrm{t}) \mathrm{t}}(\ln \mathrm{wh})+\sum_{j=3}^{6} \beta_{\mathrm{j}} \mathrm{DK}_{\mathrm{i}(\mathrm{t}) \mathrm{t}}+\sum_{k=1}^{12} \delta_{\mathrm{k}} \mathrm{ce}_{\mathrm{i}(\mathrm{t})} \\
& +\sum_{t=1}^{8} \varphi_{\mathrm{t}} \gamma_{\mathrm{t}}+\zeta \lambda_{\mathrm{i}(\mathrm{t}) \mathrm{t}}+\varepsilon_{\mathrm{i}(\mathrm{t}) \mathrm{t}}
\end{aligned}
$$

Finalmente, incluiu-se a variável renda domiciliar entre os regressores, conforme expresso na equação (23). Se essa variável for significativa, o valor do lazer é afetado pela renda familiar corrente, mesmo mantendo-se a utilidade marginal da renda constante. Nesse caso, há evidências de que restrição à liquidez é um fenômeno importante e de que a utilidade não é perfeitamente suavizada ao longo do ciclo de vida. A fim de tornar os 
resultados comparáveis aos obtidos por Blundell et al. (1998) para o Reino Unido, optou-se por utilizar o número de horas como variável dependente, em vez do logaritmo de horas trabalhadas.

$$
\begin{aligned}
& \mathrm{h}_{\mathrm{i}(\mathrm{t}) \mathrm{t}}=\beta_{0}+\beta_{1} \ln \mathrm{w}_{\mathrm{i}(\mathrm{t}) \mathrm{t}}+\beta_{2} \mathrm{y}_{\mathrm{i}(\mathrm{t}) \mathrm{t}}+\beta_{3} \mathrm{v}_{\mathrm{i}(\mathrm{t}) \mathrm{t}}^{(\ln \mathrm{w})}+\beta_{4} \mathrm{v}_{\mathrm{i}(\mathrm{t}) \mathrm{t}}(\ln \mathrm{y}) \\
& +\sum_{j=5}^{8} \beta_{\mathrm{j}} \mathrm{DK}_{\mathrm{i}(\mathrm{t}) \mathrm{t}}+\sum_{k=1}^{12} \delta_{\mathrm{k}} \mathrm{ce}_{\mathrm{i}(\mathrm{t})}+\sum_{t=1}^{8} \varphi \gamma_{\mathrm{t}}+\xi \lambda_{\mathrm{i}(\mathrm{t}) \mathrm{t}}+\varepsilon_{\mathrm{i}(\mathrm{t}) \mathrm{t}}
\end{aligned}
$$

Na equação acima, $\mathrm{y}_{\mathrm{i}(\mathrm{t}) \mathrm{t}}$ é a renda domiciliar, excluindo-se a renda da mulher, e $\mathrm{v}_{\mathrm{i}(\mathrm{t}) \mathrm{t}}(\ln \mathrm{y})$ é o correspondente resíduo. As demais variáveis são definidas como anteriormente.

\section{IV.2 Estimação}

A técnica de adição de resíduos mencionada anteriormente foi utilizada para se estimar o modelo proposto acima. Num primeiro estágio, regrediramse as variáveis endógenas (salário, logaritmo do salário, renda familiar e logaritmo da renda familiar) contra dummies de coorte e educação, tempo e interações entre coorte, educação e tempo apenas para as mulheres que ofertam um número de horas superior a zero. Em seguida, incluíram-se os resíduos calculados nesse estágio entre os regressores das equações (20) a (23), também estimadas com base na parcela da amostra que trabalha.

Com relação à razão de Mills invertida, ela foi calculada previamente por meio da estimação de um probit para toda a amostra, objetivando modelar a decisão de trabalhar em função de dummies de coorte e educação, tempo e interações entre coorte, educação e tempo.

\section{IV.3 Resultados Empiricos}

A seguir, serão apresentados os principais resultados obtidos a partir das estimativas dos modelos de oferta de trabalho abordados neste estudo. $\mathrm{Na}$ Tabela 4, os coeficientes reportados, bem como as estatísticas calculadas, referem-se aos modelos (20)-(23), estimados segundo o procedimento 
descrito na seção anterior. Abaixo de cada coeficiente pode-se observar os respectivos desvios padrões.

TABELA 4 - OFERTA DE TRABALHO NO BRASIL - VARIÁVEIS INSTRUMENTAIS

\begin{tabular}{|c|c|c|c|c|}
\hline Modelo & Stone-Geary & $\begin{array}{l}\text { Blundell- } \\
\text { Duncan- } \\
\text { Meghir }\end{array}$ & $\begin{array}{l}\text { Heckman- } \\
\text { MaCurdy (22) }\end{array}$ & $\begin{array}{c}\text { Heckman- } \\
\text { MaCurdy (23) }\end{array}$ \\
\hline \multirow[t]{2}{*}{ Constante } & & 48,0122 & 3,9876 & 3,2503 \\
\hline & & $(2,8635)$ & 0,0761 & 0,2043 \\
\hline \multirow[t]{2}{*}{ DK 0-2 } & $-1,0078$ & $(-1,6105)$ & $-0,0486$ & $-0,0908$ \\
\hline & 2,7813 & 0,4202 & 0,0121 & 0,0116 \\
\hline \multirow[t]{2}{*}{ DK 3-4 } & $-4,7508$ & $(-2,9849)$ & $-0,1071$ & $-0,0693$ \\
\hline & 2,0341 & 0,4432 & 0,0106 & 0,0128 \\
\hline \multirow[t]{2}{*}{ DK 5-10 } & $-4,2631$ & $-2,8267$ & $-0,1096$ & $-0,0413$ \\
\hline & 1,9168 & 0,4612 & 0.0111 & 0,0132 \\
\hline \multirow[t]{2}{*}{ DK 11+ } & $-2,9669$ & $-1,5761$ & $-0,0595$ & $-0,0069$ \\
\hline & 1,6108 & 0,3183 & 0,0085 & 0,0092 \\
\hline \multirow[t]{2}{*}{ Salário } & 31,5871 & & & \\
\hline & $(1,7558)$ & & & \\
\hline \multirow[t]{2}{*}{ Logaritmo do Salário } & & $-6,9724$ & $-0,2132$ & \\
\hline & & $(1,1572)$ & $(0,0371)$ & \\
\hline \multirow[t]{2}{*}{ Renda do Domicílio } & & 0,0008 & & \\
\hline & & $(0,0008)$ & & \\
\hline \multirow[t]{2}{*}{ Logaritmo da Renda } & & & & 0,0479 \\
\hline & & & & $(0,0380)$ \\
\hline \multicolumn{5}{|l|}{ Resíduos } \\
\hline \multirow[t]{2}{*}{ Salário } & $-13,9509$ & & & \\
\hline & $(1,7562)$ & & & \\
\hline \multirow[t]{2}{*}{ Logaritmo do Salário } & & 2,8285 & 0,0827 & \\
\hline & & $(1,1573)$ & $(0,0371)$ & \\
\hline \multirow[t]{2}{*}{ Renda do Domicílio } & & $-0,00004$ & & \\
\hline & & $(0,0008)$ & & \\
\hline \multirow[t]{2}{*}{ Logaritmo da Renda } & & & & 0,1265 \\
\hline & & & & $(0,0380)$ \\
\hline \multirow[t]{2}{*}{ Participação } & $-23,9494$ & $-11,9760$ & $-0,4980$ & 0,0095 \\
\hline & 15,6665 & 2,1971 & 0,0668 & 0,0708 \\
\hline
\end{tabular}

Nota: Desvios padrões entre parêntesis. 
Com o intuito de verificar as diferenças entre o método de pseudopainel e as regressões feitas por meio de mínimos quadrados ordinários, expõem-se abaixo as estimativas correspondentes aos modelos (20)-(23) obtidas por meio desse último estimador. É importante ressaltar que, na obtenção dessas estimativas, não foram incluídas nas equações principais interações entre educação e coorte e resíduos para controlar a endogeneidade das variáveis e o viés de seleção.

TABELA 5 - OFERTA DE TRABALHO NO BRASIL - MÍNIMOS QUADRADOS ORDINÁRIOS

\begin{tabular}{lcccc}
\hline Modelo & $\begin{array}{c}\text { Stone- } \\
\text { Geary }\end{array}$ & $\begin{array}{l}\text { Blundell- } \\
\text { Duncan- } \\
\text { Meghir }\end{array}$ & $\begin{array}{c}\text { Heckman- } \\
\text { MaCurdy (21) }\end{array}$ & $\begin{array}{r}\text { Heckman- } \\
\text { MaCurdy (22) }\end{array}$ \\
\hline Constante & & 37,8611 & 3.5642 & 2.8881 \\
DK 0-2 & 7.6633 & $-2,3101$ & -0.0900 & $(0.0048)$ \\
& $(1.0509)$ & $(0,1363)$ & $(0.0050)$ & -0.0419 \\
DK 3-4 & 3.3072 & $-2,0771$ & -0.0826 & $(0.0048)$ \\
& 1.1206 & 0,1442 & 0.0053 & -0.0194 \\
DK 5-10 & 5.5136 & $-1,3962$ & -0.0605 & 0.0051 \\
DK 11+ & 0.8105 & 0,1102 & 0.0040 & -0.0037 \\
Salário & 8.8159 & $-0,7543$ & -0.0302 & 0.0039 \\
& 0.9550 & 0,1243 & 0.0045 & 0.0110 \\
Logaritmo do & 18.6997 & & & 0.0044 \\
Salário & $(0.0501)$ & & & \\
Renda do Domicílio & & $-3,3478$ & & \\
Logaritmo da Renda & & $(-0,0428)$ & $(0.0014)$ & \\
& & 0,0007 & & \\
\hline
\end{tabular}

Nota: Desvios padrões entre parêntesis.

O resultado da comparação das estimativas depende muito do modelo escolhido. Quando se estima o modelo de Stone-Geary por mínimos quadrados ordinários, o coeficiente correspondente ao salário sofre uma redução de 
quase $50 \%$ e as variáveis demográficas passam a apresentar coeficientes positivos e significativamente diferentes de 0 , contrariamente ao esperado.

Já no modelo de Blundell-Duncan-Meghir, observa-se que o coeficiente do logaritmo do salário, independentemente do método de estimação utilizado, apresenta um sinal negativo, contrário ao previsto pela teoria econômica. $\mathrm{O}$ valor absoluto do coeficiente mais que dobra quando se estima o modelo pelo método de pseudopainéis. Mesmo com controles para endogeneidade das variáveis e viés de seleção, a renda familiar manteve um sinal positivo, também contrário ao esperado, embora não significativo e bastante reduzido. As variáveis demográficas em ambos os casos sugerem que a presença de filhos reduz a quantidade de horas ofertada no mercado de trabalho.

Por fim, cabe destacar que, no modelo de Heckman-MaCurdy, quando se utiliza o método de pseudopainéis, os coeficientes associados ao logaritmo do salário e ao logaritmo da renda sofrem uma redução. No primeiro caso, passa de $-0,0935$ para $-0,2132$, apresentando novamente um sinal oposto ao esperado. Já no segundo, passa de 0.1538 para 0.1265 . Por outro lado, os coeficientes das variáveis demográficas, embora significativos em alguns casos, são bastante próximos de 0 quando estimados tanto por mínimos quadrados ordinários quanto pelo método de pseudopainéis.

As Tabelas 6 e 7 apresentam as elasticidades calculadas para as variáveis relevantes de cada modelo. ${ }^{17}$

\section{TABELA 6 - ELASTICIDADES DE OFERTA DE TRABALHO - VARIÁ- VEIS INSTRUMENTAIS}

\begin{tabular}{lcccc}
\hline Modelo & Stone-Geary & Blundell & Heckman-MaCurdy(22) & Heckman-MaCurdy(23) \\
\hline Elasticidades & $-0,134$ & $-0,191$ & $-0,213$ & 0,050 \\
\hline
\end{tabular}

17 No modelo de Stone-Geary, expresso na equação (20), a elasticidade é dada por $\beta_{1} / \bar{h}-1$. 
TABELA 7 - ELASTICIDADES DE OFERTA DE TRABALHO - MQO

\begin{tabular}{lcccc}
\hline Modelo & Stone-Geary & Blundell & Heckman-MaCurdy(22) & Heckman-MaCurdy(23) \\
\hline Elasticidades & $-0,487$ & $-0,092$ & $-0,093$ & 0,182 \\
\hline
\end{tabular}

Levando-se em conta as elasticidades calculadas por meio do método de Variáveis Instrumentais (Tabela 6), pode-se perceber que as mesmas encontram-se entre -0.213 e 0,050 , o que significa uma sensibilidade moderada das horas de trabalho ofertadas pelas mulheres com relação a variações no salário principal.

Considerando-se as estimativas obtidas por OLS, observa-se uma maior discrepância entre as elasticidades dos diversos modelos, que passam a estar compreendidas entre -0.487 e 0.182 (Tabela 7 ). Mesmo sem controles para endogeneidade e viés de seleção, os sinais das elasticidades permanecem inalterados.

\section{CONCLUSÃO}

Esse artigo objetivou investigar a magnitude e o sinal da resposta do número de horas ofertado pelas mulheres com relação a alterações no salário, controlando-se pela endogeneidade dos regressores e pelo viés de seleção. Levando-se em conta os resultados como um todo, pode-se dizer que a estimação dos parâmetros correspondeu à análise preliminar de correlação no que se refere à principal variável de determinação do número de horas ofertadas pelas mulheres no mercado de trabalho: o salário real das mesmas. Entretanto, deve-se ressaltar que as elasticidades negativas encontradas não são consistentes com os modelos teóricos tradicionais.

Seria conveniente comparar estas elasticidades com estudos anteriores que utilizaram metodologias semelhantes. Angrist (1991), por exemplo, utilizando dados da PSID de 1969 a 1979, encontrou uma elasticidade negativa de $-0,336$ para o modelo de Stone-Geary e de -0,132 e 0,0032, respectivamente, para as equaçóes de Heckman-MaCurdy com o logaritmo do salário e o logaritmo da renda como variáveis dependentes. Dois fatores, 
entretanto, diferenciam ambos os trabalhos. Primeiramente, Angrist estima os dados agrupando as variáveis somente por ano. Além disso, não trata da questão do viés de seleção. Essa pode ser uma das causas das diferenças nas estimativas. Os resultados obtidos por Blundell et al.(1998), utilizando dados do Reino Unido entre 1978 a 1991, indicam elasticidades compreendidas entre 0,140 e 0,173 .

É interessante sublinhar algumas questões que não foram tratadas neste trabalho e que podem ser objeto de pesquisa futura. Primeiramente, testes mais conclusivos a respeito da validade dos instrumentos e da exclusão da equação principal das interações entre coorte, educação e tempo podem ser levados a cabo seguindo novamente a metodologia empregada por Blundell et al. (1998). Um segundo aspecto que pode ser incorporado é a inclusão de impostos. No caso de serem progressivos, sua presença reduz a elasticidade da oferta de trabalho.

\section{APÊNDICE}

Derivação da Equação (3)

O problema do consumidor é maximizar

$$
\sum_{t} \frac{1}{(1+\rho)^{t}}\left[B_{1} \ln \left(\gamma-\mathrm{h}_{\mathrm{t}}\right)+B_{2} \ln \left(\mathrm{c}_{\mathrm{t}}-\xi\right)\right]
$$

sujeito a

$$
\sum_{t} \frac{1}{(1+r)^{t}}\left[\mathrm{w}_{\mathrm{t}} \mathrm{h}_{\mathrm{t}}+\mathrm{y}_{\mathrm{t}}-\mathrm{c}_{\mathrm{t}}\right]=0
$$

O lagrangiano é dado por:

$\mathrm{L}=\sum_{t} \frac{1}{(1+\rho)^{t}}\left[B_{1} \ln \left(\gamma-\mathrm{h}_{\mathrm{t}}\right)+B_{2} \ln \left(\mathrm{c}_{\mathrm{t}}-\xi\right)\right]+\lambda_{0} \sum_{t} \frac{1}{(1+r)^{t}}\left[\mathrm{w}_{\mathrm{t}} \mathrm{h}_{\mathrm{t}}+\mathrm{y}_{\mathrm{t}}-\mathrm{c}_{\mathrm{t}}\right](\mathrm{A} 1)$ 
Diferenciando com relação a $h_{t}, c_{t}$ e $\lambda_{0}$, obtemos as seguintes condições necessárias de primeira ordem para maximização:

$$
\begin{aligned}
& \frac{\partial L}{\partial h_{t}}=0=-\frac{1}{(1+\rho)^{t}} B_{1} \frac{1}{\gamma-h_{t}}+\lambda_{0} \frac{1}{(1+r)^{t}} \mathrm{w}_{\mathrm{t}}=0=\frac{B_{1}}{(1+\rho)^{t}} \frac{1}{\gamma-h_{t}}=\lambda_{0} \frac{1}{(1+r)^{t}} \mathrm{w}_{\mathrm{t}} \\
& \Rightarrow \frac{B_{i t}^{*}}{\gamma-h_{t}}=\lambda_{0 \mathrm{w}_{\mathrm{t}}}{ }^{*}, \quad B_{1 t} *=B_{1}(1+\rho)^{-\mathrm{t}} \mathrm{e} \quad \mathrm{w}_{\mathrm{t}}^{*}=\mathrm{w}_{\mathrm{t}}(1+\mathrm{r})^{-\mathrm{t}} \\
& \frac{\partial L}{\partial c_{t}}=0 \Rightarrow \frac{1}{(1+\rho)^{t}} B_{2} \frac{1}{c_{t}-\zeta}-\lambda_{0} \frac{1}{(1+r)^{t}}=0=>\frac{B_{2}}{(1+\rho)^{t}} \frac{1}{c_{t}-\zeta}=\lambda_{0} \frac{1}{(1+r)^{t}} \\
& \Rightarrow \frac{B_{2 t}^{*}}{c_{t}-\zeta}=\lambda_{0} \mathrm{p}_{\mathrm{t}}^{*}, \quad B_{2 t} * B_{2}(1+\rho)^{-\mathrm{t}} \mathrm{e} \mathrm{p}_{\mathrm{t}}^{*}=(1+\mathrm{r})^{-\mathrm{t}} \\
& \frac{\partial L}{\partial \lambda_{0}}=0=>\sum_{t} \frac{1}{(1+r)^{t}}\left[\mathrm{w}_{\mathrm{t}} \mathrm{h}_{\mathrm{t}}+\mathrm{y}_{\mathrm{t}}-\mathrm{c}_{\mathrm{t}}\right]=0
\end{aligned}
$$

Resolvendo (A2) e (A3) para $B_{i} *$ e somando com relação a t, temos que:

$$
\begin{gathered}
\sum_{t}\left(B_{1 t} *+B_{2 t} *\right)=\lambda_{0} \sum_{t} \frac{1}{(1+r)^{t}}\left[\mathrm{w}_{\mathrm{t}} \gamma \mathrm{w}_{\mathrm{t}} \mathrm{h}_{\mathrm{t}}+\mathrm{c}_{\mathrm{t}}-\zeta\right] \\
\sum_{t}\left(B_{1 t} *+B_{2 t} *\right)=\lambda_{0} \sum_{t} \frac{1}{(1+r)^{t}}\left[\mathrm{w}_{\mathrm{t}} \gamma+\mathrm{y}_{\mathrm{t}}-\zeta\right], \Rightarrow \\
\lambda_{0}=\sum_{t} \frac{\left(B_{1 t} *+B_{2 t} *\right)}{F^{*}}, \mathrm{~F}^{*}=\sum_{t}\left[\mathrm{w}_{\mathrm{t}} * \gamma+\mathrm{y}_{\mathrm{t}} * \mathrm{p}_{\mathrm{t}} * \zeta\right] \text { e } \mathrm{y}_{\mathrm{t}}^{*}=\mathrm{y}_{\mathrm{t}}(1+\mathrm{r})^{-\mathrm{t}}
\end{gathered}
$$

Finalmente, substituindo (A5) em (A2):

$$
\begin{aligned}
& \frac{B_{i t}^{*}}{\gamma-h_{t}}=\sum_{t} \frac{\left(B_{1 t}^{*}+B_{2 t}^{*}\right)}{\mathrm{F}^{*}} \mathrm{w}_{\mathrm{t}}^{*}=>\mathrm{w}_{\mathrm{t}} * \mathrm{~h}_{\mathrm{t}}-\mathrm{w}_{\mathrm{t}}^{*} \gamma=-\frac{B_{1 t} *}{\sum_{t}\left(B_{1 t} *+B_{2 t} *\right)} \mathrm{F}^{*}=> \\
& \mathrm{w}_{\mathrm{t}} \mathrm{h}_{\mathrm{t}}^{*}=\gamma \mathrm{w}_{\mathrm{t}}-\left(\frac{1+r}{1+\rho}\right)^{t} \tilde{B}_{1} F^{*}=>\mathrm{w}_{\mathrm{t}} \mathrm{h}_{\mathrm{t}}=\gamma \mathrm{w}_{\mathrm{t}}-\eta^{t} \tilde{B}_{1} F^{*}, \\
& \eta=\frac{1+r}{1+\rho} \text { e } \tilde{B}_{1}=\frac{B_{1}}{\sum_{t}\left(B_{1 t} * B_{2 t} *\right)}
\end{aligned}
$$


Derivação das Equações (10a), (10b), (11) e (12)

O problema do consumidor é maximizar:

$\sum_{o}^{T} \frac{1}{(1+\rho)^{t}}\left[\mathrm{~A}(\mathrm{t})(\mathrm{L}(\mathrm{t}))^{\alpha}+\mathrm{B}(\mathrm{t})(\mathrm{C}(\mathrm{t}))^{\beta}\right]$

sujeito a

$\mathrm{y}_{\mathrm{t}}=\sum_{o}^{T} \frac{1}{(1+r)^{t}}[\mathrm{C}(\mathrm{t})-\mathrm{W}(\mathrm{t})(1-\mathrm{L}(\mathrm{t}))]$ e $0 \leq \mathrm{L}(\mathrm{t}) \leq \bar{L}$

O lagrangiano é dado por:

$\Pi=\sum_{o}^{T} \frac{1}{(1+\rho)^{t}}\left[\mathrm{~A}(\mathrm{t})(\mathrm{L}(\mathrm{t}))^{\alpha}+\mathrm{B}(\mathrm{t})(\mathrm{C}(\mathrm{t}))^{\beta}\right]-\lambda_{0}\left\{\sum_{o}^{T} \frac{1}{(1+r)^{t}}[\mathrm{C}(\mathrm{t})-\mathrm{W}(\mathrm{t})(1-\mathrm{L}(\mathrm{t}))]-\mathrm{y}_{\mathrm{t}}\right\}$

Diferenciando com relação a L(t), obtém-se:

$\frac{\partial \Pi}{\partial L(t)}=0=>\frac{\alpha}{(1+\rho)^{t}} \mathrm{~A}(\mathrm{t})(\mathrm{L}(\mathrm{t}))^{\alpha-1}-\lambda_{0} \frac{1}{(1+r)^{t}} \mathrm{~W}(\mathrm{t})=0$

Resolvendo (A8) para L(t) tem-se que:

$\mathrm{L}(\mathrm{t})=\left[\frac{1}{A(t) \alpha}\left(\frac{1+\rho}{1+r}\right)^{t} \lambda(0) W(t)\right]^{\frac{1}{\alpha-1}}$

se o indivíduo trabalha.

Aplicando logaritmo a ambos os lados de (A9), utilizando o fato de que $\mathrm{L}(\mathrm{t})=\bar{L}-\mathrm{h}(\mathrm{t})$ e aproximando $\ln [(\mathrm{l}+\rho) /(\mathrm{l}+\mathrm{r})]$ por $\rho-\mathrm{r}$, tem-se que:

$\ln (\bar{L}-\mathrm{h}(\mathrm{t}))=1 /(\alpha-1)[\ln \lambda(0)-\ln \alpha+(\rho-\mathrm{r}) \mathrm{t}-\ln \mathrm{A}(\mathrm{t})+\ln \mathrm{W}(\mathrm{t})] \quad(\mathrm{A} 10)$

Somando-se $1 /(1-\alpha) \ln [\bar{L}-\mathrm{h}(\mathrm{t})]$ a ambos os lados de (A10) e dividindo -se por $\alpha /(\alpha-1)$ chega-se a (All): 
$\ln (\bar{L}-\mathrm{h}(\mathrm{t}))=1 / \alpha[\ln \lambda(0)-\ln \alpha+(\rho-\mathrm{r}) \mathrm{t}-\ln \mathrm{A}(\mathrm{t})+\ln \mathrm{W}(\mathrm{t}) \mathrm{h}(\mathrm{t})]$

Procedimento Utilizado para a Construção da Amostra

A amostra utilizada neste trabalho consiste de todas as observações da Pesquisa Nacional por Amostra de Domicílios (PNAD) dos anos de 1992, 1993, 1995-99, excetuando-se os indivíduos:

i) do sexo masculino;

ii) com idade inferior a 24 ou superior a 55 anos;

iii)que não ocupam a condição de cônjuge na unidade domiciliar;

iv) que se ignora se trabalharam na semana de referência;

v) cujo número de horas trabalhadas na semana de referência é ignorado;

vi) cujo ano de nascimento do último filho nascido vivo é ignorado ou o quesito não se aplica;

vii) cujo último filho nascido vivo está morto;

viii) para os quais não se conhece o número de anos de estudo;

ix) cujo valor do rendimento mensal de todos os trabalhos é ignorado ou não aplicável;

x) cujo valor do rendimento mensal familiar é ignorado ou não aplicável;

xi) que trabalharam na semana de referência, mas cujo rendimento mensal de todos os trabalhos é 0 ;

\section{BIBLIOGRAFIA}

ANGRIST, J. Grouped data estimation and testing in simple labor-supply models. Journal of Econometrics, v. 47, p. 243-266, 1991. 
ASHENFELTER, O.; HAM, J. Education, unemployment and earnings. Journal of Political Economy, v. 87, n. 5, p. 99-116, 1979.

BLUNDELL, R.; DUNCAN, A.; MEGHIR, C. Estimating labour supply responses using tax reforms. Econometrica, v. 66, n. 4, p. 827-861, 1998.

BROWNING, M.; DEATON, A.; IRISH, M. A profitable approach to labor supply and commodity demands over the life-cycle. Econometrica, v. 53 , n. 3 , p. 503-543, 1985.

GREENE, W. H. Econometric analysis. $3^{\text {rd }}$ Edition. New Jersey: Prentice Hall. 1997.

HECKMAN, J. Sample selection bias as a specification error. Econometrica, v. 47, n. 1, p. 153-161, 1979.

HECKMAN, J.; MACURDY, T. A life-cycle model of female labor supply. Review of Economic Studies, v. 47, p. 47-74, 1980.

JATOBA, J. A família brasileira na força de trabalho: um estudo de oferta de trabalho - 1978/88. Pesquisa e Planejamento Econômico, v. 24, n. 1, 1994.

MACURDY, T. An empirical model of labor supply in a life cycle setting. Journal of Political Economy, 89, p. 1059-1086, 1981.

MOFFITT, R. Identification and estimation of dynamic models with a time series of repeated cross-sections. Journal of Econometrics, v. 59, p. 99-123, 1993.

MROZ, T. The sensitivity of an empirical model of married women's hours of work to economic and statistical assumptions. Econometrica, v. 55, n. 4, p. 765-799, 1987.

RIBEIRO, E. Asymmetric labor supply. Empirical Economics, v. 26, n. 1, 2000.

SEDLACEK, G.; SANTOS, E. A mulher cônjuge no mercado de trabalho como estratégia de geração da renda familiar. Pesquisa e Planejamento Econômico, v. 21, n. 3, 1991.

(Recebido em outubro de 2001. Aceito para publicação em julho de 2003). 